\title{
Impedance Spectroscopy Measurements for Gotten Membrane and Membrane-Receptor, in the Presence of Bisphenol A
}

\author{
Andrés Ernesto Mejía, Chad Leidy, María T. Cortés \\ Universidad de Los Andes, Calle 25 A \#4-76 apto 201, 2432460 Colombia
}

Received 25 April 2008; accepted 15 February 2009

\begin{abstract}
The adverse effects of endocrine disruptors on human health have created a need for screening systems to detect xenoestrogens. In this project, we develop a system to detect one of these xenestrogens, Bisphenol A (BPA), through the development of a biosensor based on impedance measurements. The biosensor consists of a supported lipid bilayer that incorporates a protein receptor sensitive to the presence of BPA. This setup was originally proposed by V. Granek and J. Rishpon [19]; we now present an alternative method of analysis to increase sensibility. As a preliminary evaluation of bioactivity of BPA we measured the direct interaction of this xenoestrogen with the membrane by analyzing changes in the membrane impedance as a function of BPA concentration. The results indicate that BPA inserts and disrupts the membrane, but only at very high unphysiological BPA concentrations ( $>1 \mathrm{ppm}$ ). We then developed a high sensitivity biosensor, based on the detection of the electric signal by means of impedance spectroscopy, resulting from the interaction between BPA and an estrogen receptor reconstituted in a lipid membrane. The results show that the presence of the receptor increases by several orders of magnitude the sensitivity of the system, making it possible to detect BPA at ppb.
\end{abstract}

Keywords: electrochemical measures in hormonal range, biosensor, impedance spectroscopy, Bisphenol A, membrane, receptor, xenoestrogen, endocrine disruptors.

\section{Introducción}

Los estrógenos son hormonas sexuales producidos por los ovarios y en pequeñas cantidades por la glándula adrenal y los testículos [1]. Inducen fenómenos de proliferación celular sobre diversos órganos, principalmente órganos sexuales de carácter femenino, como el endometrio, mama y el propio ovario. Actúan sobre

\footnotetext{
* Corresponding author. E-mail address: andre-me@uniandes.edu.co, andreme21@gmail.com
} 
diversos grupos celulares del organismo, especialmente con algunos relacionados con la actividad sexual; también con el cerebro, en donde presentan función endocrina y neurotransmisora. En su función endocrina, los estrógenos atraviesan la membrana celular para llegar al núcleo, en el que se encargan de activar o desactivar determinados genes, regulando la síntesis de proteínas[1]. Los xenoestrógenos son sustancias externas a los organismos vivos, que al ingresar son capaces de simular las actividades de las hormonas naturales causando desórdenes fisiológicos. Por tanto, también se denominan de manera alternativa como disruptores endocrinos [2, 3]. Las sustancias xenoestrógenas han comenzado a incorporarse sistemáticamente al medio ambiente de manera relativamente reciente (menos de 70 años), a través de la industria química en general [2, 3]. Varias investigaciones recientes han mostrado que los xenoestrógenos pueden ser, en gran medida, responsables por la preocupante disminución en la cantidad y calidad del esperma humano, observada en los últimos 50 años $[4,5]$. Por lo tanto es importante desarrollar técnicas sensibles, específicas y eficientes, para la detección de estos componentes.

El Bisfenol A (BPA), un xenoestrógeno, es un compuesto aromático tipo fenol que se obtiene por la reacción del fenol con la acetona, en relación de 2 a 1. Esta sustancia fue sintetizada, por primera vez, en 1891 por Dianin [6]. Actualmente, su principal uso es como monómero en la síntesis de poli carbonato y resinas epóxicas, así como antioxidante e inhibidor de la polimerización en el PVC [7]. La migración al ser humano fue descrita con el uso de plásticos de policarbonato en los equipos de hemodiálisis [8]. La alta afinidad del BPA con las proteínas receptoras de estrógeno, lo cual altera su funcionamiento normal, causan daños fisiológicos que no pueden ser reparados fácilmente. Nagel mostró que la exposición de ratones hembra en estado fetal a dosis de BPA, en el rango típico de exposición de los humanos, altera el crecimiento postnatal y genera una pubertad temprana en ratones hembra [9-11]. La cromatografía, ensayos biológicos, sensores químicos y biosensores son los métodos comúnmente usados para la determinación de BPA [12-19].

\section{Descripción del proyecto \\ Hipótesis}

El propósito global de esta propuesta es el desarrollo de un método alternativo a la cromatografía, para la detección de BPA en disoluciones acuosas. Concretamente, el desarrollo de un biosensor basado en receptores específicos incorporados a una membrana lipídica apoyada sobre un electrodo y cuya señal de detección es una medida de la resistividad del sistema. La propuesta se basa en estudios previos que han demostrado la efectividad de la espectroscopia de impedancia electroquímica para detectar cambios en la resistividad de un electrodo, en cuya superficie se depositan moléculas de Bisfenol A [20]. La membrana lipídica permite la incorporación de un receptor específico para la molécula de interés. Por tanto, se propone el desarrollo de un biosensor basado en una metodología sencilla y altamente sensible para la detección de BPA. 


\section{Materiales}

El 1-palmitoyl y el 2-oleoylphosphatidylcholine (POPC) fueron adquiridos por medio de Avanti Polar Lipids (Alabaster, AL). Este lípido presenta una transición de fase gel/fluido a $-5^{\circ} \mathrm{C}$, lo cual garantiza que la membrana se mantenga en fase fluida durante la elaboración de la membrana apoyada y durante las medidas. El Decanetiol y el Bisfenol A fueron adquiridos en Sigma-Aldrich (St. Louis, MS). El receptor de estrógeno recombinante (alfa) fue adquirido en Calbiochem (La Jolla, CA). Todos los reactivos adicionales fueron de calidad cromatografía y fueron adquiridos en Sigma-Aldrich (St. Louis, MS)

Para facilitar la limpieza del tiol descrita en una sección posterior, se usaron como electrodos láminas de platino y de oro de alta pureza. Se utilizó una lámina de platino de $0,75 \mathrm{~mm}$ de espesor con un área superficial eficaz de deposición de la membrana de $1 \mathrm{~cm}^{2}$. Adicionalmente, se utilizo una lámina de oro de $0,71 \mathrm{~mm}$ de espesor, con área eficaz de deposición de la membrana de $1 \mathrm{~cm}^{2}$, adquiridas a través de un distribuidor local. Para la limpieza y pulido de los electrodos se usaron emulsiones de diamante (gruesa de $15 \mu \mathrm{m}$, media de $6 \mu \mathrm{m}$ y fina de 1 $\mu \mathrm{m}$ ); el material de pulido fue adquirido en Bioanalytical Systems (Lafayette, IN).

\section{Metodología}

\section{Formación y caracterización}

Por medidas de impedancias, de una membrana lipídica apoyada sobre electrodos de oro y de platino, en presencia de BPA.

Generación de una membrana lipídica sobre electrodos metálicos

Limpieza de los electrodos

Los electrodos requieren una limpieza, para eliminar óxidos y residuos orgánicos, previa a la toma de medidas. La limpieza consta de un método abrasivo y una limpieza electroquímica. Se pulen los electrodos, haciendo uso de emulsiones de diamante de tamaños 15,6 y $1 \mu \mathrm{m}$, y posteriormente se enjuagan con etanol en baño de ultrasonidos durante 10 minutos, para desprender de la superficie de trabajo partículas adheridas. Por último se procede a un pulido fino con una disolución de alfa alúmina de $0,5 \mu \mathrm{m}$ durante 10 minutos en baño de ultrasonido. Una vez realizada la limpieza abrasiva, se procede a la limpieza electroquímica en disolución de $\mathrm{NaOH} 0,1$ M mediante una voltametría cíclica (30 ciclos)

Preparación de la membrana apoyada

Los lípidos permanecen en la nevera a $-20{ }^{\circ} \mathrm{C}$ para evitar su degradación. Antes de la preparación de las muestras, los lípidos se colocan en un desecador durante 20 minutos para evitar problemas de condensación durante el proceso de descongelación. En la balanza de precisión se pesan las cantidades apropiadas de los lípidos y los contenedores se devuelven a la nevera inmediatamente. Los lípidos se hidratan en agua nanopura, cometiéndolos a vortex durante 10 minutos para dar una concentración final de $0,2 \mathrm{mM}$ de POPC. Esta disolución se introduce en un baño de ultrasonidos hasta obtener una disolución transparente 
(aproximadamente durante 15 minutos), lo cual indica la presencia de vesículas unilamelares pequeñas, de un diámetro promedio de $50 \mathrm{~nm}$.

La deposición se hace sumergiendo el electrodo de platino o de oro, limpio y tratado con decanatiol, en la disolución de vesículas durante $1 \mathrm{hr}$. Se cambia la disolución varias veces para eliminar el exceso de vesículas, de acuerdo con el siguiente procedimiento: se realiza un primer enjuague, para eliminar las vesículas sobrantes, con $20 \mathrm{~mL}$ de $\mathrm{PBS}$ (pH 7,4); se eliminan $5 \mathrm{~mL}$ de la disolución y se realiza un segundo enjuague con $15 \mathrm{~mL}$, finalmente se eliminan 5 $\mathrm{mL}$ resultando una disolución de $10 \mathrm{~mL}$.

La membrana queda así lista para llevar a cabo el análisis electroquímico en el que se mide la impedancia para cerciorarnos de la formación de una membrana apoyada en la superficie del electrodo (Fig. 1).

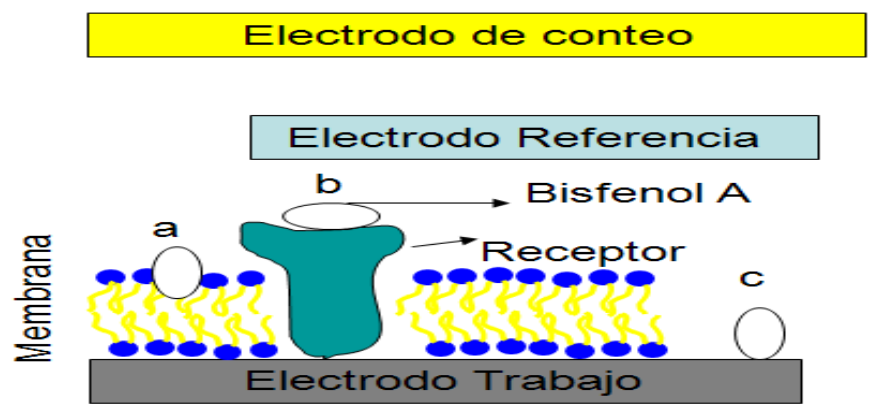

Figura 1. Diagrama de montaje del biosensor.

Metodología del procedimiento de medida de impedancias

Condiciones del potenciostato para la realización de los análisis

Se realizan barridos de frecuencias desde $30000 \mathrm{~Hz}$ hasta $100 \mathrm{mHz}$,_con amplitud de $10 \mathrm{mV}$ centrada en $170 \mathrm{mV} \mathrm{CC} \mathrm{para} \mathrm{platino} \mathrm{y}-120 \mathrm{mV}$ para Au. Estos rangos de operación óptimos en voltaje, se determinaron al analizar regiones de corriente constante para una variación de $15 \mathrm{mV}$, tanto para polarización como para despolarización, sobre la curva de voltametría cíclica.

Estudio del comportamiento electroquímico de los sistemas lipídicos (membrana -receptor) por espectroscopia de impedancias (EIS) en disolución acuosa

Se propone un circuito equivalente que se ajuste al sistema lipídico con el mínimo número de elementos, en base al número de polos observados a partir de los diagramas BODE del espectro de impedancias (Fig. 2) y se hace un seguimiento de los cambios en los parámetros del circuito por la adición de BPA. Con la información obtenida en esta etapa, se verifica el circuito equivalente propuesto buscando el mejor ajuste a esos parámetros. Este análisis permitirá deducir una relación entre la concentración de BPA añadida a la disolución y alguno de los elementos que caracterizan el circuito. 


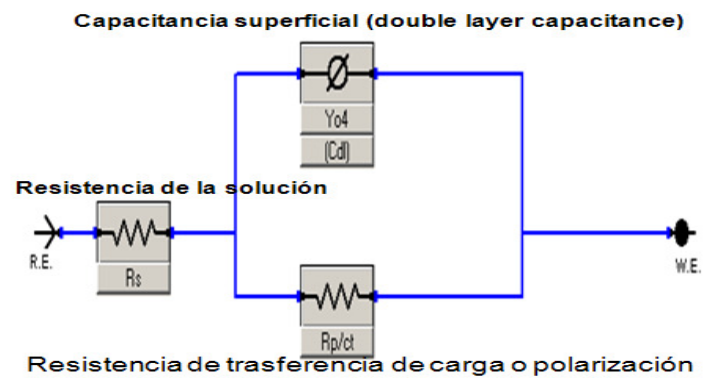

Figura 2a. Modelo de circuito equivalente para el platino.

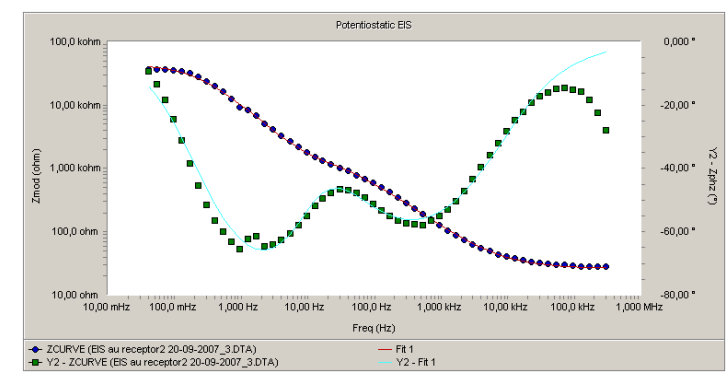

Figura 2c. Diagrama de Bode y ajuste con el modelo oro y tiol.

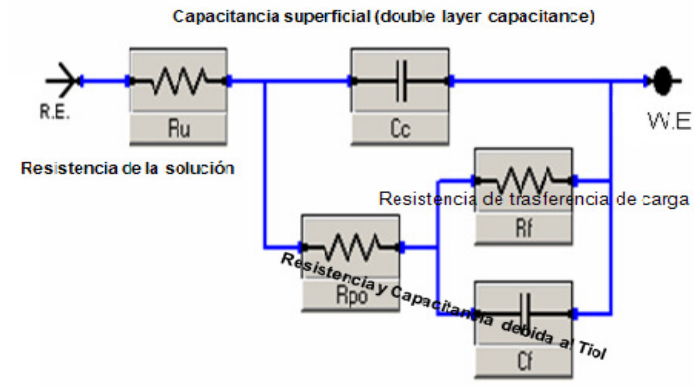

Figura 2b. Modelo de circuito equivalente para el electrodo oro y tiol.

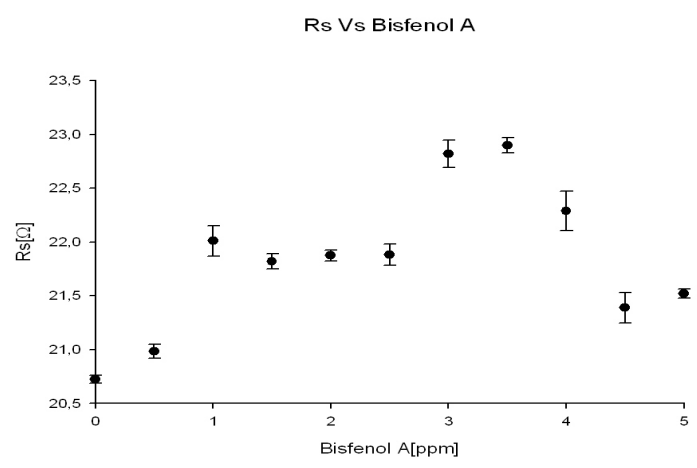

Figura 2d. Se observa que en el modelo propuesto la variación de la resistencia de la disolución, Rs vs. BPA, varía entre 20,7 y 23 $\Omega$.

\section{Resultados}

En la Fig. 1 se muestra un diagrama del montaje del biosensor sobre platino. Proponemos que el BPA presenta principalmente dos procesos por los que puede generar un cambio en la respuesta de impedancia: interactuando con la membrana directamente (Fig. 1a) y generando un cambio estructural en la membrana condicionado por el receptor (Fig. 1b). Las Fig. 2a y b muestran los circuitos equivalentes que hemos ajustado para platino y oro, respectivamente, buscando caracterizarlos a través de parámetros sencillos y que dieran una buena respuesta vía Fitting (Fig 2c). En la Fig. 2d se observa que a partir de los modelos propuestos, con y sin membrana para platino y oro, la resistencia de la disolución (Rs y $\mathrm{Ru}$ de las Figs. 2a y 2 b) no varía apreciablemente con el incremento de BPA. Podemos inferir que el BPA se adhiere preferentemente al electrodo en los rangos de concentración utilizados. Esto verifica la validez del método usado para detectar pequeñas cantidades de BPA y también permite enfocar nuestra atención solo hacia los efectos de superficie del electrodo. 


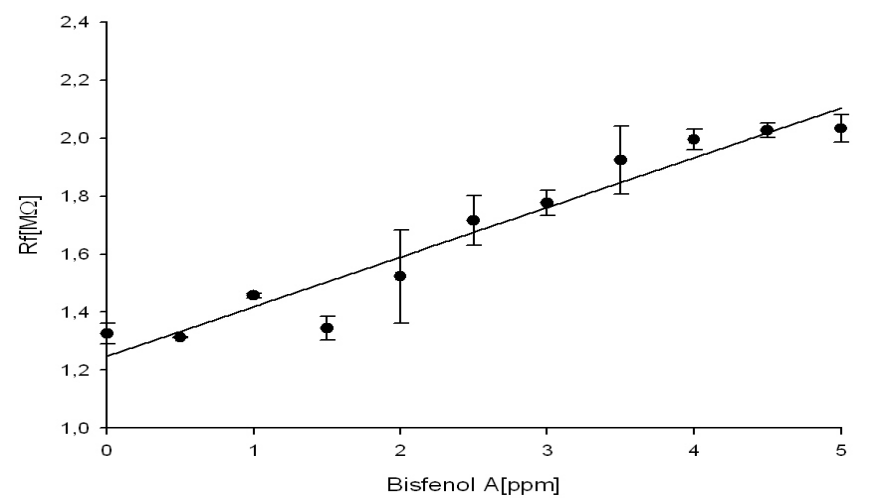

Figura 3. Comportamiento del parámetro $\mathrm{R}_{\mathrm{f}}$ (electrodo de oro) para el caso con membrana, al incrementar la concentración de BPA.

El electrodo de oro presenta una respuesta de impedancia más compleja que la del platino, lo que requiere más elementos para su adecuado modelado. Esta complejidad surge por la presencia de decanatiol en la superficie. Tanto Rs como Yo se mantienen aproximadamente constantes frente a la presencia del BPA; esto indica que la variación de la resistencia de la disolución, así como de la capacitancia de la membrana y capacitancia de interacción de BPA-electrodo de platino o poros, se mantiene con pocas variaciones en este modelo. La incorporación de BPA es por tanto detectada a través de variaciones en la resistencia de membrana $\left(R_{f}\right)$, presentada en la Fig. 3. En dicha Fig. 3 se observa que la variación de la resistencia es lineal y ocurre en el rango de partes por millón (ppm). Estos datos indican que el BPA puede interactuar directamente con la membrana lipídica en ausencia del receptor de estrógeno, pero los efectos sobre la membrana son solo detectables en concentraciones no fisiológicas, alrededor de tres órdenes de magnitud superiores a las reportadas a nivel ambiental.

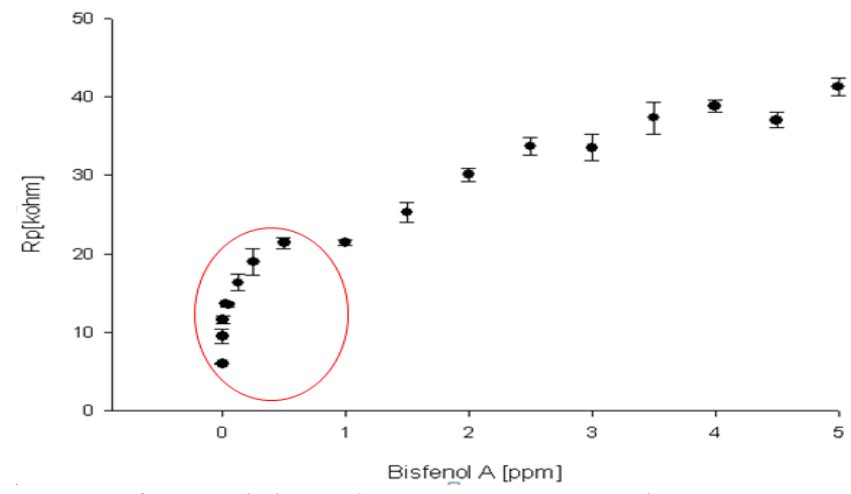

Figura 4. Comportamiento del parámetro Rp, para el caso con receptor vs. BPA.

La segunda etapa de este proyecto se enfocó a investigar si la incorporación de receptores de estrógeno a la membrana apoyada proporcionaba un rango más amplio de sensibilidad para la detección de BPA y proveía de selectividad al método. Evaluamos la respuesta de Pt y Au en presencia de membrana y RE a niveles crecientes de BPA. Esperábamos que el receptor actuara como 
transductor de señal, relacionando la adhesión de BPA al sitio activo del receptor, con un cambio en las propiedades físicas de la membrana y, por ende, a una respuesta electroquímica detectable (Fig. 4). El receptor presenta una alta afinidad por el BPA. Bajo condiciones estándar $\left(22^{\circ} \mathrm{C}\right.$ y en incubación durante 2 horas) 1 unidad de receptor adhiere 1 pmol de $3 \mathrm{H}$-estradiol, lo que equivale a 0,335 moléculas de estradiol por receptor. Para la preparación de muestras, se adicionaron 10 unidades de receptor por muestra durante el periodo de incubación (30 pmoles de proteína por muestra). Al tomar en cuenta que tenemos un volumen por muestra de $10 \mathrm{~mL}$ y asumiendo que el $100 \%$ del receptor se adhiere a la membrana durante el periodo de incubación, el límite de saturación en la detección de BPA, exclusivamente por adhesión con receptores, seria de $\sim 2,3 \mathrm{ppm}$ (asumiendo que el BPA y el estradiol presentan el mismo comportamiento de adhesión al receptor). Estas concentraciones, aunque aproximadas, sugieren que el rango de detección en base a receptores, debe encontrarse en el rango de ppb de BPA y que se debe presentar una saturación de receptores en rangos de ppm. Esto se observa claramente en los datos presentados a continuación. Para el caso del oro a baja concentración (menor de 0,5 ppm de BPA) no se observó una tendencia clara en los resultados (datos no mostrados). Por otro lado, obtuvimos una respuesta clara con el sistema receptor/membrana en base al electrodo de platino (Fig. 4). Se observa una curva de saturación en el rango de partes por billón, que llega a un punto de saturación antes de $1 \mathrm{ppm}$. Interpretamos el comportamiento general de la curva mostrada en la Fig. 4, como una saturación a bajas concentraciones del receptor, el cual presenta alta afinidad por el BPA, seguido de la incorporación de BPA directamente en la membrana observada. Es decir, la superposición de los procesos (a) y (b) descrito en la Fig. 1. Se analizaron otros compuestos, presentando estructuras moleculares similares al BPA, para evaluar si el sistema de detección en base al receptor era especifico al BPA. Los datos demuestran que el receptor genera una respuesta en el rango de ppb, lo que indica que el receptor no responde específicamente al BPA. Los dos compuestos que se evaluaron fueron nonilfenol y fenol (Fig. 5).

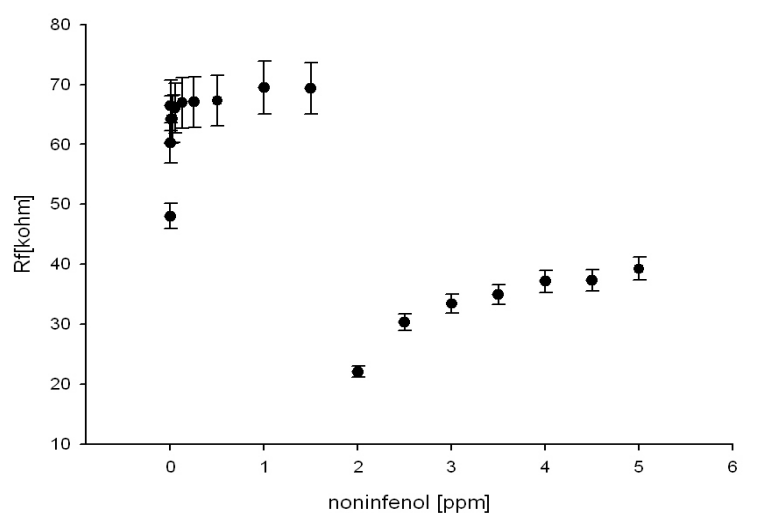

Figura 5. Comportamiento del parámetro Rp, para el caso con receptor vs. noninfenol. 


\section{Conclusiones}

Se ha estandarizado el protocolo para la deposición de una membrana sobre electrodos de platino y de oro y se ha observado que la membrana es sensible a la presencia de BPA en concentraciones superiores a $1 \mathrm{ppm}$. El aumento de la resistencia de polarización de ambos modelos en presencia de BPA, indica una interacción directa entre el BPA y la membrana.

Se ha logrado generar un sistema altamente sensible con Pt, membrana y receptor, que presenta sensibilidad en el rango de $10 \mathrm{ppb}$, que es el rango hormonal. Sin embargo, el sistema resultó no ser específico al BPA debido a que el biosensor respondió en estos rangos a la incorporación de fenol y nonilfenol. La respuesta a estos tres compuestos indica que el biosensor desarrollado en este trabajo actúa como detector general de compuestos que puedan actuar como disruptores del receptor de estrógeno y que el sistema, sin un análisis más profundo, no puede utilizarse para la detección especifica. Por lo tanto, el sistema tiene potencial como método de detección de diferentes compuestos que puedan actuar como xenoestrógenos, al interactuar directamente con el receptor de estrógeno.

\section{Medidas de Espectroscopia de Impedancia sobre membrana y membrana-receptor apoyadas, en presencia de Bisfenol A}

\section{Resumen}

Los efectos adversos de los disruptores endocrinos (DE) sobre la salud, crean la necesidad de generar sistemas de detección de xenoestrógenos (DE). En este proyecto desarrollamos un sistema para la detección de uno de estos xenoestrógenos, el Bisfenol A (BPA), a través de la construcción de un biosensor basado en medidas de espectroscopia de impedancia para mejorar la sensibilidad. El biosensor consta de un soporte (electrodo) y una doble capa lipídica a la que le incorporamos una proteína receptor de estrógenos. Esta idea fue inicialmente concebida por V. Granek y J. Rishpon (Detecting Endocrine-Disrupting Compounds by Fast Impedance Measurements, Environ. Sci. Technol. 36 (2002) 1574-1578). Realizamos una evaluación de la bioactividad de BPA con la membrana, a través del análisis de cambios en la impedancia en función de la concentración de BPA. Los resultados encontrados indicaron que el BPA cambia la configuración de la membrana, pero en concentraciones no fisiológicas $(>1 \mathrm{ppm})$. Posteriormente, con la incorporación del receptor de estrógeno sobre la membrana lipídica, desarrollamos un biosensor de mayor sensibilidad haciendo posible la detección de BPA en ppb.

Palavras clave: medidas electroquímicas en rango hormonal, biosensor, espectroscopia de impedancia, Bisfenol A, membrana, receptor, xenoestrógeno, disruptores endocrinos.

\section{Referencias}

1. Nussey and Whitehead, Endocrinology, an integrated approach, Taylor and Francis 2001.

2. J.J. Amaral Mendes, The endocrine disrupters: a major medical challenge, 
Food and Chemical Toxicology 40 (2002) 781-788.

3. A. Johnson, Endocrine active industrial chemicals: release and occurrence in the environment, Pure Appl. Chem. 75 (2003) 1895-1904.

4. F. Eertmans, W. Dhooge, S. Stuyvaert, F. Comhaire, Endocrine disruptors: effects on male fertility and screening tools for their assessment, Toxicology in Vitro 17 (2003) 515-524.

5. O. Tsutsumi, Assessment of human contamination of estrogenic endocrinedisrupting chemicals and their risk for human reproduction, The Journal of Steroid Biochemistry and Molecular Biology 93 (2005) 325-330.

6. Dianin, Zhurnal russkogo fiziko-khimicheskogo obshchestva 23 (1891) 492.

7. K.L. Howdeshell, P.H. Peterman, B.M. Judy, J.A. Taylor, C.E. Orazio, R.L. Ruhlen, F.S.V. Saal, W.V. Welshons, Environ. Health Perspect. 111(2003) 1180-1187.

8. H. Yamasaki, Y. Nagako, H. Makino, Nephron 88 (2001) 376-378.

9. S.C. Nagel, F.S.V. Saal, W.V. Welshons, Environ. Health Perspect 105 (1997) 70-76.

10. S.C. Nagel, F.S.V. Saal, W.V. Welshons, J. Steroid Biochem. Molec. Biol. 69 (1999) 343-357.

11. K.L. Howdeshell, A.K. Hotchkiss, K.A. Thayer, J.G. Vanderbergh, F.S.V Saal, Nature 401 (1999) 763-764.

12. A.J. Oosterkamp, B. Hock, M. Seifert, H. Irth, Novel monitoring strategies for xenoestrogens, Trends in Analytical Chemistry 16 (1997) 544-553.

13. X. Wang, H. Zeng, L. Zhao, J.-M. Lin, Selective determination of bisphenol A (BPA) in water by a reversible fluorescence sensor using pyrene/dimethyl [beta]-cyclodextrin complex, Analytica Chimica Acta 556 (2006) 313-318.

14. X. Ye, Z. Kuklenyik, L.L. Needham, A.M. Calafat, Automated On-Line Column-Switching HPLC-MS/MS Method with Peak Focusing for the Determination of Nine Environmental Phenols in Urine, Anal. Chem. 77 (2005) 5407-5413.

15. J.D. Stuart, C.P. Capulong, K.D. Launer, X. Pan, Analyses of phenolic endocrine disrupting chemicals in marine samples by both gas and liquid chromatography-mass spectrometry, Journal of Chromatography A 1079 (2005) 136-145.

16. J.L. Vilchez, A. Zafra, A. Gonzalez-Casado, E. Hontoria, M. del Olmo, Determination of trace amounts of bisphenol F, bisphenol $\mathrm{A}$ and their diglycidyl ethers in wastewater by gas chromatography-mass spectrometry, Analytica Chimica Acta 431 (2001) 31-40.

17. S. Rodriguez-Mozaz, M.L. de Alda, D. Barcelo, Analysis of bisphenol A in natural waters by means of an optical immunosensor, Water Research 39 (2005) 5071-5079.

18. M. Holger, B. Karlheinz, Determination of Endocrine-Disrupting Phenolic Compounds and Estrogens in Surface and Drinking Water by HRGC(NCI)-MS in the Picogram per Liter Range, Environ. Sci. Technol. 35 (2001) 3201-3206.

19. V. Granek, J. Rishpon, Detecting Endocrine-Disrupting Compounds by Fast Impedance Measurements, Environ. Sci. Technol. 36 (2002) 1574-1578. 
20. M.A. Méndez, M.F. Suárez, M.T. Cortés, Electrochemical impedance spectroscopy of diluted solutions of Bisphenol A, Journal of Electroanalytical Chemistry. In press (2006). 